\title{
An Optimized algorithm for device discovery using BLE
}

\author{
Yan Zhang ${ }^{1, \text { a }}$, Zheng Song ${ }^{2}$, Wendong Wang ${ }^{1}$, Ye Tian ${ }^{1}$ \\ ${ }^{1}$ State Key Laboratory of Networking and Switching Technology, Beijing University \\ Of Posts and Telecommunications, Beijing, China \\ ${ }^{2}$ Software Innovation Lab, Virginia Tech, Blacksburg, U.S.A \\ azhangyan_kxmyt@163.com
}

Keywords: device discovery, BLE, Android.

\begin{abstract}
There are more and more mobile smart devices with high capabilities in both computing and storage, which leading to the device cooperation in vicinity becomes popular. As the first step in the device cooperation, the device discovery process is particularly important. Because of the mobility of the devices and the limitation of the energy, how to perform the device discovery process efficiently is the key of the device cooperation. However, the devices support not only one type of wireless communication modules, choose which module to perform the device discovery is the first issue to think about. This paper compares the existing wireless communication technologies and find the suitable method. Then proposes an optimized algorithm for the device discovery using Bluetooth Low Energy (BLE).
\end{abstract}

\section{Introduction}

Mobile smart devices are more and more popular, such as smart phones, smart watches and so on. And the capabilities of mobile smart devices have been greatly improved: such as computing capability, storage capacity, making the smart devices not only as client to request services from the server, but can serve as server to provide appropriate services. This leads to the popularity of smart device cooperation in vicinity.

Owing to the device discovery process is the first step in device cooperation, so it is especially important. The current mobile devices support a variety of wireless network communication interfaces. Therefore, performing the device discovery process more efficiently using the suitable wireless communication technology is the first and important step.

In this paper, we compare the device discovery with various wireless communication methods and select the optimal wireless communication method firstly. Then we propose an optimized algorithm for device discovery through Bluetooth Low Energy (BLE) and do experiments to verify the algorithm finally.

\section{Related Work}

Existing wireless communication technologies include Bluetooth/BLE [1], [2], WiFi/WiFiP2P [3], NFC [4], Zigbee [5], etc. However, in most Android, iPhone, and other smart devices, only NFC, WiFi/WiFiP2P and Bluetooth/BLE modules are mounted.

Owing to the range of the nearby devices cooperation is about tens of meters, which makes the corresponding wireless communication technologies should communicate among tens of meters. According to the research of NFC [4], the communication range of NFC is less than or equal to 0.1 meters, and the traditional Bluetooth is about 10 meters. The limitation of communication range lead to the fact that NFC and Bluetooth are not suitable for device discovery in nearby device cooperation.

As the communication range of BLE and WiFi is nearly 100 meters, they are both suitable for device discovery process. As is introduced in [6], WiFi/WiFiP2P have higher data transmission rate, but consume more energy per unit working time. On the other hand, BLE consume less energy in unit time, but have much lower data transmission rate [7]. [8] and [9] propose an eDiscovery 
algorithm to using the BLE to find the nearby devices. However, this algorithm can not deal the problem when the number of the devices scanned is same. Based on this, we propose an optimized algorithm to suit both the number of devices scanned is same or different.

\section{Device discovery}

\subsection{BLE and WiFi for device discovery}

From the above section, we can get the differences of BLE and WiFi wireless technologies. Owing to there are no need to transfer larger data in the device discovery process, which makes the transfer speed is not essential. That's, the BLE wireless communication technology have great advantage in device discovery.

In addition to the perceptual comparison of the two wireless communication technologies, we also verify the advantage of BLE in energy consumption by doing experiments.

Before comparing BLE and WiFi, we first analyze the process of device discovery using two wireless communication technologies. In order to make the description simpler, RD represents the request device in the device cooperation, and $\mathrm{AD}$ represents the assist device.

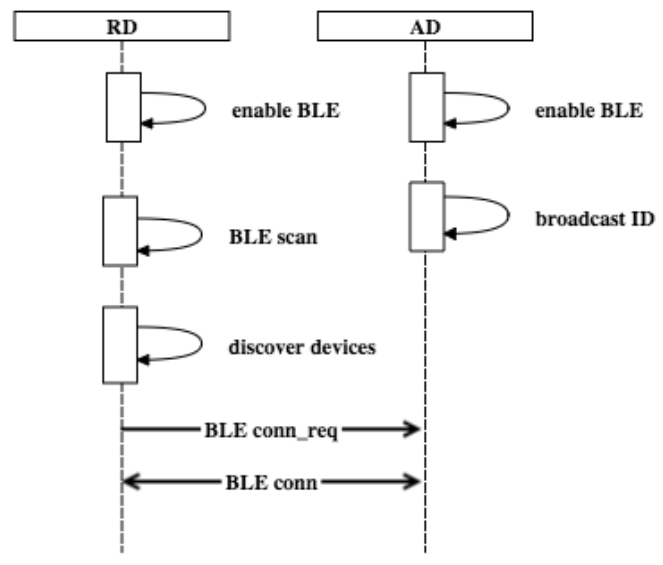

a) BLE

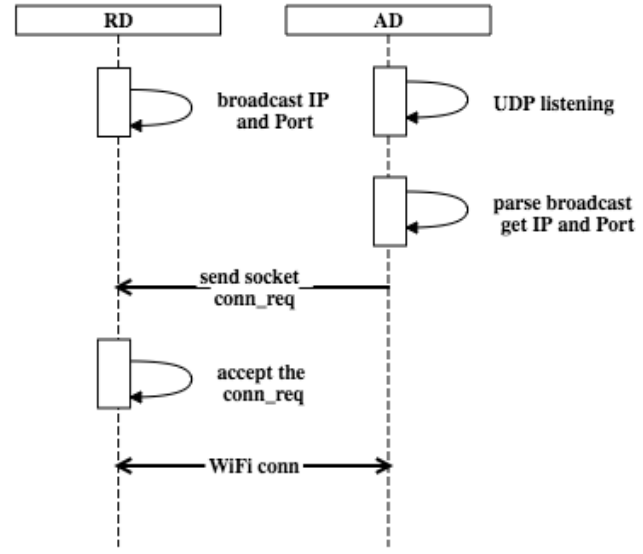

b) WiFi

Fig.1 the process of device discovery using BLE or WiFi

The figure 1 shows the process of the device discovery using BLE and WiFi.

1) BLE: the AD needs to be in the peripheral mode to broadcast their own ID information, the $\mathrm{RD}$ should in the central mode to scan the AD by ID at the same time. Then the devices can establish the BLE connection between RD and AD.

2) WiFi: the RD needs to send UDP broadcast information which includes its own IP and port. On the other hand, the AD needs to listen to the UDP broadcast information so as to obtain the IP and port. Then the $\mathrm{AD}$ sends socket connection request and establish the WiFi socket connection after the $\mathrm{RD}$ accept the request.

Here, we verify the BLE wireless technology by analyzing the energy consumption of the AD, since the RD is the initiator of the nearby device cooperation. And we do experiments on BLE and WiFi using the above procedure.

In order to exclude other factors on the experimental result, we set the initial battery is $100 \%$, transfer the screen to the darkest and execute for four hours respectively.

The results show that the comparative experiment consumes $13.5 \%$ battery, the BLE broadcast consumes $15 \%$ and the UDP listening consumes 18\%. From the data we can get the advantage in energy consumption of BLE. So, we use BLE in our device discovery process. 


\subsection{Device cooperation in vicinity}

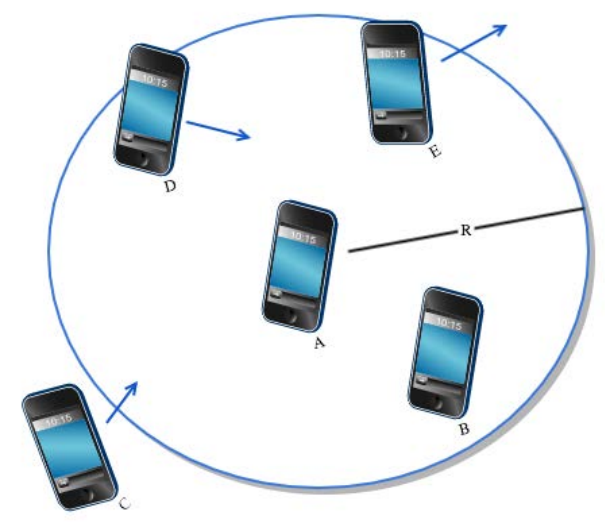

Fig.2 The scene graph of device cooperation in vicinity.

In the figure 2, the device $\mathrm{A}$ is as the $\mathrm{RD}$, the other devices are as $\mathrm{ADs}$, and the cooperation range is $\mathrm{R}$. We can see, the mobility is the significant feature in the nearby device cooperation, and the mobility influence the accuracy of the discovered devices.

This paper is mainly from the perspective of the $\mathrm{RD}$, so as to design the optimized device discovery algorithm for the nearby device cooperation aiming at balancing the accuracy and energy consumption.

\subsection{Optimized device discovery algorithm}

In order to get the most precise nearby devices with less energy, we provide the follow schemes.

1) constant scheme: the RD will scan the nearby devices constantly;

2) Fixed interval scheme: the RD will at fixed interval to scan, and scan time is fixed;

As for the above schemes, the constant scheme can guarantee the accuracy of the nearby devices' mobility with high energy consumption. Opposite of this, the fixed interval scheme can save energy with lower accuracy.

In order to balance the accuracy and energy consumption, we propose our optimized algorithm for the device discovery. The core idea of the algorithm is to dynamically adjust the scan time and scan interval according to two consecutive scan results, in order to ensure accuracy and energy consumption.

Table 1 List of notation

\begin{tabular}{|c|c|}
\hline interval $_{\text {init }}$ & Scan interval assignment \\
\hline duration $_{\text {init }}$ & Scan duration assignment \\
\hline interval $_{i}$ & The i-th scan interval \\
\hline duration $_{i}$ & The i-th scan duration \\
\hline $\begin{array}{l}r \\
\text { time }_{\max }\end{array}$ & $\begin{array}{l}\text { Similarity coefficient of two adjacent scans } \\
\text { The max value of scan interval or duration }\end{array}$ \\
\hline time $_{\text {min }}$ & The min value of scan interval or duration \\
\hline $\mathrm{Num}_{i}$ & The device number of i-th scan \\
\hline List $_{i}$ & The device list of i-th scan \\
\hline$R$ & The cooperation range \\
\hline segment & The max value for interval \\
\hline
\end{tabular}

Owing to the correlation coefficient $r$ is designed to get the intervali +1 and durationi +1 , getting the accurate $r$ is the key to the algorithm.

In principle, comparing the two adjacent scan results to modify the interval and scan duration of the next time is the most precise method. However, the process of compare the adjacent scan results is energy-consuming and time-consuming, so, it will make a big overhead in the device discovery 
process. Taking the above issue into account, we compare the number of devices discovered of the two adjacent scan firstly. Once the number is same, we will do the next step: compare the device listss of the two adjacent scan. And this can reduce the times of comparing the two adjacent scan results.

And the optimized algorithm is as below:

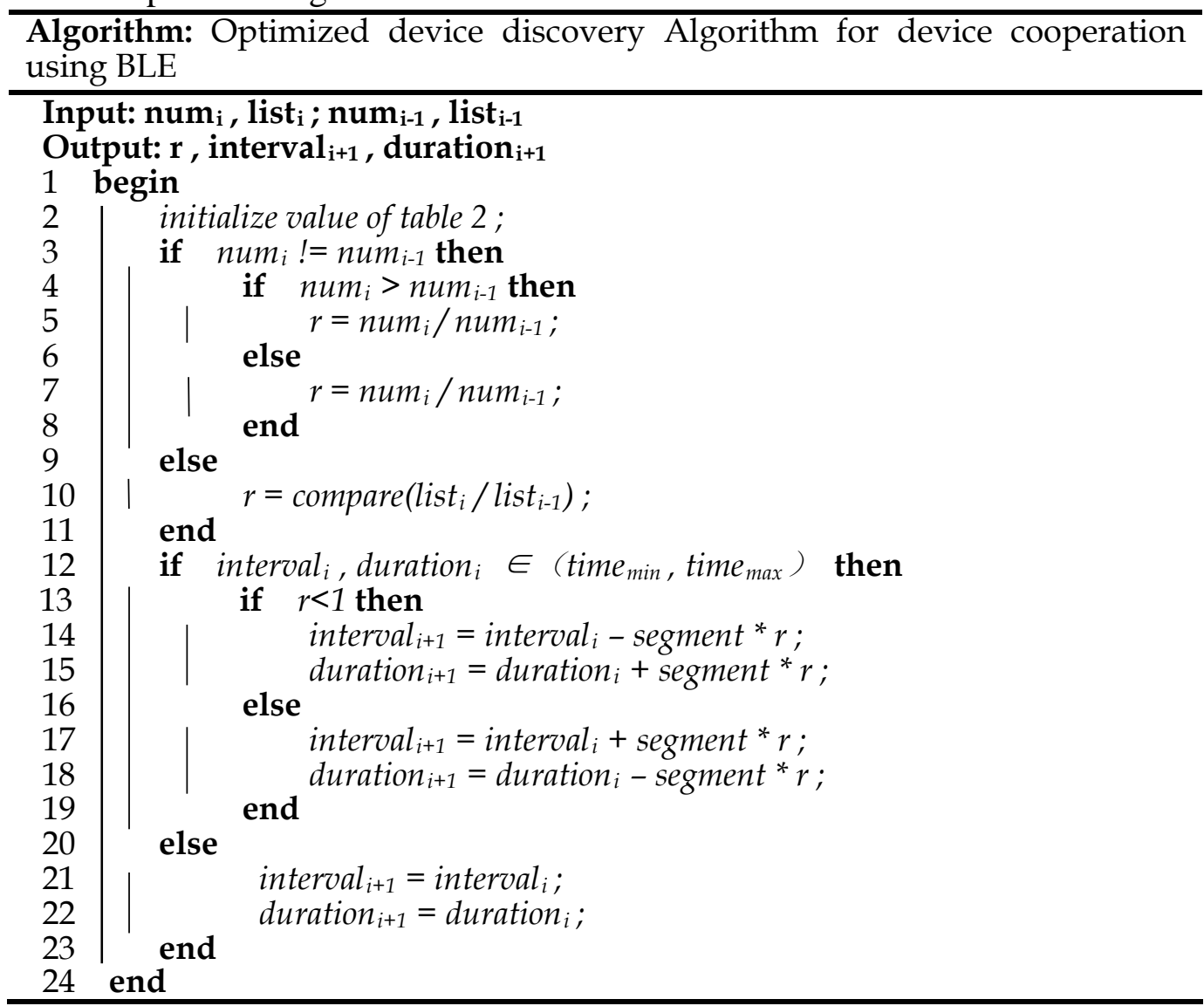

\section{Implementation and Experiment}

We implement the optimized device discovery algorithm on Android platform using BLE and implement the constant and fixed interval algorithm as well to verify the optimized algorithm.

In order to verify the feasibility of the algorithm, we use the following devices: two Nexus6, a Nexus9, and two iphone. Nexus9 is served as RD, and the remaining devices are as ADs. Except this, we make the battery of Nexus 9 is $100 \%$ and the brightness is the same in each experiment. Four hours later, we will get the data generated in each experiment. We will analyze the data in the two aspects: energy-consumption and the accuracy of the device discovery.

\subsection{Static scenario}

The static scenario contains two cases: 1) all devices are in a relatively static state; 2) the devices moves in the range which the RD can discover. From the data, we can find the scan duration time of optimized algorithm is decreased much than the first two algorithms. But the energy consumption has not changed much. By analyzing the origin data, we can find the optimized algorithm compare the adjacent results frequently which is energy-consumption. And the results are as bellow: 

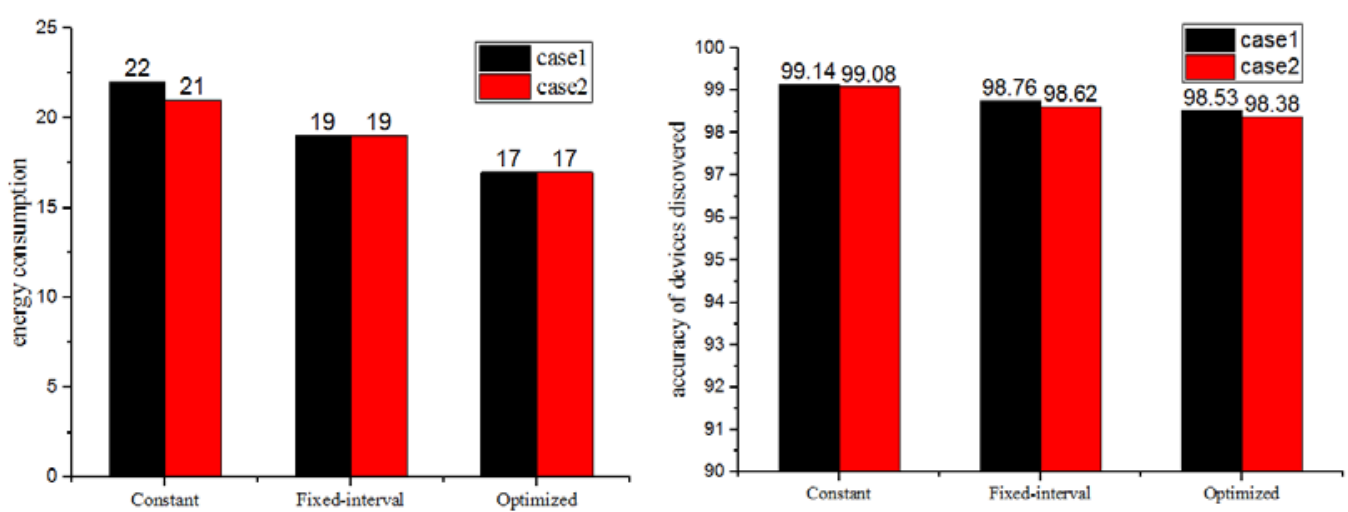

Fig.3 The results of energy consumption and accuracy in static scenario

\subsection{Dynamic scenario}

Dynamic scenario also includes two cases: 1) the total number of discovered devices equipment is the same but the devices are not the same. For example, the adjacent results are B, C, D and B, C, $\mathrm{E}$; 2) the number of the device is different.

In the dynamic environment, it is not guaranteed that the total number of devices scanned by the device at any time is always the same, and the device list is different. Therefore, the above two cases are combined to analyze the applicability of the algorithm.
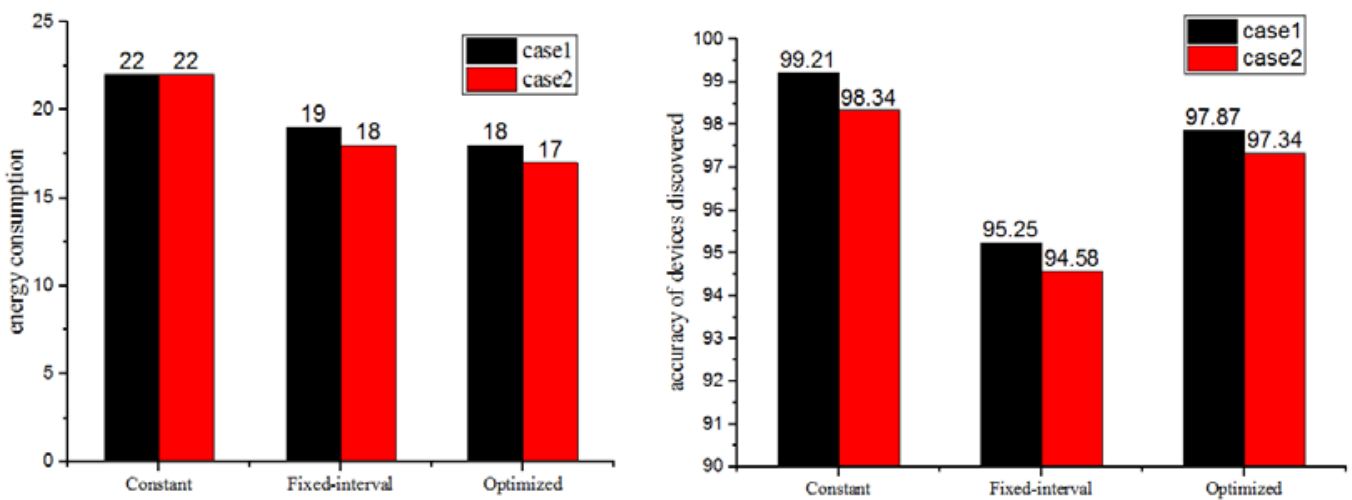

Fig.4 The results of energy consumption and accuracy in dynamic scenario

\subsection{Experiment Analysis}

In order to avoid more power consumption in the analysis of the scan results, our algorithm can solve this problem better, the device power consumption will be increased, the results are analyzed only when the current number of scans is the same, thus greatly reducing the energy consumption of each scan.

By comparing the static scenario and dynamic scenario, we can see the constant scheme is high energy-consumption with high accuracy of devices in both scenarios; the fixed interval scheme has different performance in the above two scenarios: In the static scenario, the fixed scheme is better in energy-consumption than constant scheme with slightly low accuracy. However, in the dynamic scenario, it's performance is not so good.

As for our optimized algorithm, we can get in the static scenario our algorithm has the almost same accuracy with lower energy consumption compared with fixed scheme. Except this, in the dynamic scenario, we can get our optimized algorithm has higher accuracy than the fixed scheme. And this can show that our algorithm is practical in both static or the dynamic scenarios.

\section{Summary}

This paper compares the existing wireless communication technologies and find the most suitable wireless communication method. Then proposes an optimized algorithm for the device discovery process using BLE and do the experiments to verify the algorithm in both static scenarios and dynamic scenarios. In the future, we will do the experiments in the situation which includes more devices. 


\section{Acknowledgement}

This paper is supported by the National High-tech R\&D Program of China (863 Program) under Grant No. 2015AA016101.

\section{Reference}

[1] B. Specification, “Version 1.1," See http://www. opensearch. org/Specifications/OpenSearch/1.1, 2001. iscep?

[2] C. Gomez, J. Oller, and J. Paradells, "Overview and evaluation of bluetooth low energy: An emerging low-power wireless technology,” Sensors, vol. 12, no. 9, pp. 11 734-11 753, 2012. istepp:

[3] S. Rajasekhar, I. Khalil, and Z. Tari, “A scalable and robust qos architecture for wifi p2p networks," in International Conference on Distributed Computing and Internet Technology. Springer, 2004, pp. 65-74. ișeps]

[4] R. Want, “An introduction to rfid technology,” IEEE Pervasive Comput- ing, vol. 5, no. 1, pp.

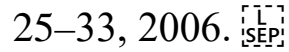

[5] Z. Alliance et al., “Zigbee specification,” 2006. i[s-p.

[6] W.-F. Alliance, "Wi-fi peer-to-peer (p2p) technical specification v1. 0,"

[7] "Specification of the bluetooth system-covered core package version: 4.0," 2010.

[8] B. Han and A. Srinivasan, "ediscovery: Energy efficient device discovery for mobile opportunistic communications,” in ICNP’12. IEEE, 2012, pp. 1-10.

[9] B. Han, J. Li, and A. Srinivasan, "On the energy efficiency of device discovery in mobile opportunistic networks: A systematic approach,” IEEE TMC, vol. 14, no. 4, pp. 786-799, 2015 